\title{
QUEEN'S
UNIVERSITY
BELFAST
}

\section{An Electric Vehicle Dispatch Module for Demand-Side Energy Participation}

Zhou, B., Yao, F., Littler, T., \& Zhang, H. (2016). An Electric Vehicle Dispatch Module for Demand-Side Energy Participation. Applied Energy, 177, 464-474. https://doi.org/10.1016/j.apenergy.2016.05.120

\author{
Published in: \\ Applied Energy
}

Document Version:

Peer reviewed version

Queen's University Belfast - Research Portal:

Link to publication record in Queen's University Belfast Research Portal

\section{Publisher rights}

2016 Elsevier Ltd. All rights reserved.

This manuscript version is made available under the CC-BY-NC-ND 4.0 license http://creativecommons.org/licenses/by-nc-nd/4.0/ which permits distribution and reproduction for non-commercial purposes, provided the author and source are cited.

\section{General rights}

Copyright for the publications made accessible via the Queen's University Belfast Research Portal is retained by the author(s) and / or other copyright owners and it is a condition of accessing these publications that users recognise and abide by the legal requirements associated with these rights.

Take down policy

The Research Portal is Queen's institutional repository that provides access to Queen's research output. Every effort has been made to ensure that content in the Research Portal does not infringe any person's rights, or applicable UK laws. If you discover content in the Research Portal that you believe breaches copyright or violates any law, please contact openaccess@qub.ac.uk. 


\title{
An Electric Vehicle Dispatch Module for Demand-Side Energy Participation
}

\author{
Bowen Zhou ${ }^{*}{ }^{1}$, Feng Yao ${ }^{1}$, Tim Littler ${ }^{1}$, Huaguang Zhang ${ }^{2}$ \\ ${ }^{1}$ School of Electronics, Electrical Engineering and Computer Science, Queen's University Belfast, Belfast, BT9 5AH, \\ UK \\ ${ }^{2}$ Electric Automation Institute, Northeastern University, Shenyang, 110004, PR China \\ bzhou02@qub.ac.uk
}

\begin{abstract}
The penetration of the electric vehicle (EV) has increased rapidly in recent years mainly as a consequence of advances in transport technology and power electronics and in response to global pressure to reduce carbon emissions and limit fossil fuel consumption. It is widely acknowledged that inappropriate provision and dispatch of EV charging can lead to negative impacts on power system infrastructure. This paper considers EV requirements and proposes a module which uses owner participation, through mobile phone apps and on-board diagnostics II (OBD-II), for scheduled vehicle charging. A multi-EV reference and single-EV real-time response (MRS2R) online algorithm is proposed to calculate the maximum and minimum adjustable limits of necessary capacity, which forms part of decision-making support in power system dispatch. The proposed EV dispatch module is evaluated in a case study and the influence of the mobile app, EV dispatch trending and commercial impact is explored.
\end{abstract}

Key words: electric vehicle; power system dispatch; real-time dispatch; mobile app; data security

\section{Nomenclature}

AFAP

AGC

ALAP

ATD

DSM

EOBD

EV

GA

MiTM

MRS2R

OBD

OS

PDF

PGP

PSO

SOC

SQP

TLS

V2G

$E_{B}$

$h_{E V i}(l)$

$N_{d}$

$P_{E V}(l)$

$P_{E V}^{\max }(l), P_{E V}^{\min }(l)$

$P_{E V i}^{\max }(l), P_{E V i}^{\min }(l)$ as-fast-as-possible

automatic generation control

as late as possible

available time duration

demand-side management

European on-board diagnostics

electric vehicle

genetic algorithm

man-in-the-middle

multi-EV reference and single-EV

real-time response

on-board diagnostics

operating system

probabilistic density function

pretty good privacy

particle swarm optimisation

state-of-charge

sequence quadratic programming

transport layer security

vehicle-to-grid

capacity of EV battery packs

electricity tariff of the $i$ th $\mathrm{EV}$ at time $l$

number of discrete time intervals

EV charging power at time $l$

maximum and minimum adjustable

limits of EV charging power

maximum and minimum limits of the $i$ th $\mathrm{EV}$ at time $l$

$P_{E V 0}$
$P_{E V}$
$P_{E V}^{M}(l)$
$P_{O}(l)$
$\operatorname{SOC}_{i}(l)$
$U\{\}$
$z$

initial value of EV charging power average EV charging power EV charging cost at time $l$ objective power at time $l$ real-time SOC of the $i$ th $\mathrm{EV}$ at time $l$

discrete uniform distribution objective function

\section{Introduction}

Power systems provide the infrastructure and energy conversion assets for electrical energy generation, transmission, distribution and consumption. In the absence of large-scale energy storage, supply and demand must be balanced instantaneously. With variations in generation output or loading, power and energy balance is normally achieved by automatic devices as well as power system dispatch and scheduling [1].

Power system loading can be both stochastic and periodic. However, statistical analysis can determine trends to calculate load forecasting and dispatch planning such that power output is controllable in near-real-time to follow demand patterns. A controllable power source is denoted by three output parameters: actual power, and maximum and minimum adjustable power. The actual power should be balanced with a load and the maximum and minimum limits provide an adjustable margin. In a unit-commitment or generation plan, these limits are also considered as constraints $[2,3]$. When there is a large change on the demand side and it is not possible to meet power balance by automatic generation control (AGC), system operators can issue dispatch orders based on these limits.

In response to global pressure to reduce carbon emissions and limit fossil fuel consumption, generation is increasingly derived from distributed and renewable sources - often with stochastic interconnection. Moreover, coupled with variable capacity availability, 
large-scale loading changes include projected connection of electric vehicles (EVs), which also pose particular challenges for energy system security and system dispatch [4]. Since renewable power output relies on primary energy density such as sufficient wind speed and adequate solar radiation, dispatch is capable of reducing power output when generation is abundant (although less able to augment output), which represents partial or semi-controllability. At the electricity consumption end, the use of EVs is inherently intermittent and stochastic, broadly based on driver travel patterns [5]. If EV demand is also controllable, it will provide another solution in power system dispatch to mitigate the variation in energy supply and render local capacity. Therefore, it is of significant importance to analyse EV behaviour in terms of power system dispatch.

The main contribution of this paper is based on a proposed online algorithm to calculate maximum and minimum adjustable power limits for EV dispatch decision-making support, from which an EV module for power system dispatch is developed. The power limits are derived from optimised EV demand profile, available time duration (ATD) and real-time state-of-charge (SOC) [6]. EV owners are able to participate in the dispatch procedure by flexible setting of the ATD using mobile-phone apps. The real-time SOC can be obtained through on-board diagnostics (OBD), which is further considered in the following section. This level of cooperation is broadly considered as demand-side participation. The technical merits of the paper contributions are considered timely and relevant to manufacturers - to improve the viability and acceptability of EVs, to owners - through participation inclusion, and to energy system operators - by offering a reliable calculation of accurate dispatch margin.

Since EVs are emerging as participant components for demand response, their connection and power system integration - as units for energy consumption or storage - require careful technical and societal negotiation. The work presented in this paper is therefore of particular relevance in demand-side management since it considers realistic and participatory connection of EVs, potentially in the context of emergent micro-grids, with respect to owner behaviour. This paper also presents the impact of vehicle integration on energy sources, especially the timely availability of local capacity to meet charging demand. In addition to rigorous development of a robust energy dispatch module framework, the paper considers practical issues, such as available time for charging, which influence and shape vehicle usage and which ultimately define how available energy is used to support driver needs.

The paper is organised as follows: Section 2 introduces related published work in EV characteristics, requirements for system dispatch, OBD, and ATD; Section 3 describes the EV module in a power system dispatch context; thereafter, Section 4 proposes a multi-EV reference and single-EV real-time response (MRS2R) (online) algorithm to calculate maximum and minimum adjustable limits of capacity; in Section 5, two objectives - the minimum payment and flat line, are established to test the feasibility of the proposed module in a case study; finally, the influence of the mobile app, $\mathrm{EV}$ dispatch trending and commercial impact is explored in Section 6.

\section{Related work}

This section presents a review of published literature and technologies relevant to EV behaviour in electrical energy system dispatch. Specific topics for EV integration are considered, which includes: ATD; requirements for system dispatch; and OBD. EVs are broadly being considered in energy planning; however their role in providing ancillary services (beyond operating in a discharge mode in a vehicle-to-grid capacity) is an emergent research topic. The particular issues for $\mathrm{EV}$ integration, in the work reported in this paper, concern the impact on energy dispatch and available capacity (for charging, primarily) and the role of EVs in terms of demand-side participation. Therefore, the following review considers these issues in detail in terms of a parametric context of EVs as aggregate units of substantial load (or capacity) in applied energy studies.

\subsection{EV characteristics}

$\mathrm{EV}$ behaviour can be parameterised in terms of time, location and magnitude. In previous studies, these issues are mathematically expressed in probability density functions (PDF), which characterise:

- The time parameter, which describes the probability of charging at a certain time interval and is expressed as a decision variable with respect to different kinds of optimisation objectives. It includes start time, end time, charging duration, and discharging duration. The charging [7] and discharging [8] duration are usually continuous from the start to end time. Such behaviour is considered as AFAP (as-fast-as-possible) charging or delayed ALAP (as-late-as-possible) charging [9]. If the ATD is introduced [10-12], the required duration will be much smaller than the setting duration (ATD), which leads to provision of non-continuous charging and discharging, and thus offers greater flexibility in EV charging management. This issue will be further considered in Section 2.2.

- The magnitude, which describes the level of charging power at a certain time interval and is related to the other decision variables while limited by uncertainties (in [13]), where the PDF of initial state-of-charge (SOC) is a most important consideration. There are usually two sources of data used to estimate this PDF. The first source is simulation data, such as normal distribution and Monte Carlo simulation [10] and the Markov-chain model [14], which can effectively describe some characteristics of EVs but it is generally not related to any real human behaviour, and thus the results are only suitable for theoretical analysis rather than consultancy or recommendations to industry or government. The second form of data is statistical, derived from conventional vehicles (internal combustion vehicles) $[7,8,13]$ and real $\mathrm{EV}$ fleets [15]. This is a more reliable data source which incorporates realistic travel patterns. However, 
vehicle statistics in $[7,8,13]$ are published annually and based on the discussion in [16], parameters of the PDF will vary in different regions and with respect to different weather conditions. Recorded EV data [15] can be used for post-event management rather than real-time communication and application.

- The EV location, which describes the geographic position of the connection node between an EV and the power system. With large-scale EV integration and reasonable (charging) station siting and sizing, the connection node can be effectively regarded as fixed, while magnitude and time are variable.

Therefore, several parameters are important to define EV behaviour for power system dispatch, which include start time, end time, ATD, initial SOC, power level, and connection location. Such parameters should be considered in the proposed EV module.

\subsection{Available time duration}

In most published work to date, EVs are assumed to be available all the time since the primary focus of EV study has been on the technical or economic benefits and ancillary services [7, 8, 14, 15]. However, the primary focus of EVs is on travelling from the perspective of EV owners, which means limited availability. The participation of EV owners to determine this availability has therefore been considered as the ATD setting.

Papers [10] and [11] assume that EVs are not connected when they are commuting from the home to workplace and vice versa. This is a general assumption which may not be entirely practical or accurate. However, in published work to date, a stipulated percentage of EVs are considered as unconnected. Probabilistic distributions of plug-in and plug-out time of EVs are assumed in [12]. This is feasible in simulation but is not suitable for real-time calculation which would additionally consider demand-side participation.

An EV connection node is easily obtained by node identification from the vehicle charger, whereas the ATD depends on the EV owner and is limited by initial SOC.

Using the initial SOC and charging power level at the connected charger, the minimum charging time can be calculated. If it is assumed that on an average working day, an EV is only used for $10 \%$ of available time $(90 \%$ redundant therefore) it is reasonable to assume the ATD for charging can be flexible and convenient, thereby creating economic incentives for the owner and power system provider. This assumption is explained in Fig. 1.

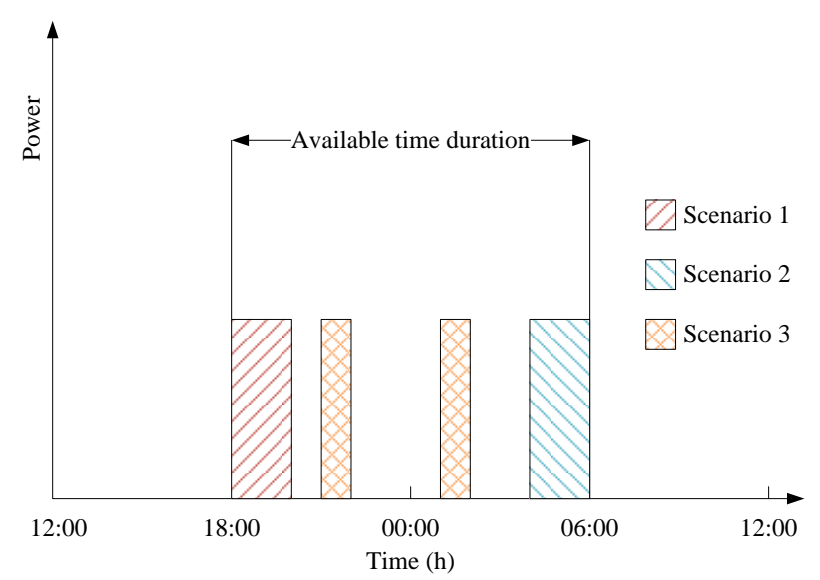

Fig. 1 Charging scenarios based on ATD

In Fig. 1 the ATD for a $24 \mathrm{~h}$ period is set from 18:00 to 6:00, meaning that people travel home in the evening and go to work the next morning. A $25 \mathrm{kWh} \mathrm{EV} \mathrm{with} \mathrm{initial}$ SOC of $56 \%$ charging at $7 \mathrm{~kW}$ power level [17] is considered. The charging profile is shown in Fig. 2. Thus, the estimated charging duration is 2 hours.

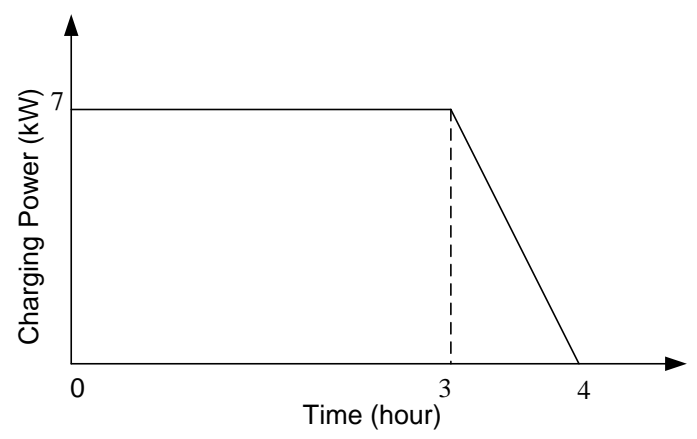

Fig. 2 Example charging profile

Since the ATD is usually much larger than the required (charging) duration, charging can commence at any time within the duration, which is described by the three scenarios in Fig. 1. Scenario 1, as in AFAP charging in [9], demonstrates that charging occurs immediately after the EV is connected and keeps charging until the EV is fully charged. This is most commonly used at present since owners prefer batteries to be fully charged and ready for any next trip as soon as possible. However, this scenario can also be regarded as irregular charging which might lead to excess loading on a power system. Scenario 1 can also be regarded as charging starting at a setting time, 00:00 for instance as off-peak charging [15]. Conversely, Scenario 2 demonstrates that an EV is fully charged, just on time and at the end of the entire duration, which can be regarded as ALAP charging in [9]. Scenario 3 demonstrates that the charging process can be non-continuous and occur any time within the entire duration.

In Northern Ireland in particular, where much of this work has been conducted, there is an operating order entitled, 'Parking Places on Roads (Electric Vehicles) (Amendment No. 3, Northern Ireland), 2014' [18], which offers a similar notation to ATD. However, it refers to the ATD of each charger, and results in limited use of the charger, which also limits flexibility of charging. The proposed ATD in this paper aims to offer a flexible basis for EV charging which limits the loading impact on a power system.

Therefore, it can be concluded that with EV owner participation (in ATD slots), there is greater flexibility for charging control, which can mean savings in terms of electricity tariff usage and thus practical operation of (EV) load control as part of demand-side management (DSM) or active energy distribution control.

To invoke owner participation, a simple mechanism would involve sending an ATD slot by mobile app to invite EV connection in a 'scheduled' dispatch. This participation will draw more engagement from EV owners and thus encourage EV uptake in a broader context. If EV dispatch is tethered to data obtained from energy markets, the financial incentives will offer further 
encouragement for participation. Although there are a few EV mobile apps available at present [19], functions are mostly a form of "navigator" to locate EV charging points as part of a travel plan. In most instances there is no interaction between a user and a server. Such apps are not feasible in an EV dispatch module since the module would require the app to send settings and receive results instantaneously. Thus, as part of this work a new mobile app was developed to offer a viable solution.

\subsection{Requirements from system dispatch}

EV charging demand is controllable, with consideration of ATD, which means it is possible to participate in dispatch scheduling and form one part of DSM. However, the maximum and minimum adjustable limits are usually not considered or assumed to be constant. This is not reasonable or practical in daily life since the EV might not be available all the time or may need to be fully charged as soon as possible, which would impact limits. Unlike units of generation, these limits cannot be constant since they change in the time domain with EV charging demand and ATD. Moreover, traditional power system dispatch is continuous in the time domain while optimal or scheduled EV charging is usually determined by discrete intervals [7, 8, 10-13]. Therefore in real-time EV dispatch, charging should include not just discrete calculation but also EV battery response when charging within the time interval.

An EV dispatch algorithm will also require a high computational efficiency and a near-real-time response. In $[20,21]$ a real-time EV charging strategy is proposed with a time interval of 1 hour: although computational efficiency is satisfied by this approach, a response is not possible within the chosen interval. Paper [11] proposes an event-driven dual-coordination mechanism for DSM of PHEVs: the mechanism is able to calculate optimal results in each time interval and a real-time response to events within the interval. The event-driven process requires involvement of all PHEVs and undertakes a three-step approach. However, it is not necessary to consider all PHEVs since the event is defined as a single PHEV charging within the interval.

\subsection{On-board diagnostics}

On-Board Diagnostics (OBD) is an automotive term referring to vehicle self-diagnostics and problem reporting capability [22]. OBD provides vehicle owners and repair technicians access to the status of various vehicle subsystems. OBD dates back to early 1980s in the USA and since then a few standard interfaces have been developed, initially used to monitor emissions. At present, the most common OBD systems are OBD-II and EOBD (European On-board Diagnostics), which is the European equivalent of OBD-II. Moreover, OBD-II and EOBD are now mandatory for all vehicles sold in the USA [23] and the European Union [24], respectively. There are also versions in other countries, for example JOBD is a version of OBD-II for vehicles sold in Japan and the ADR 79/01 (vehicle standard - Australian Design Rule 79/01 - Emission Control for Light Vehicles, 2005) is the Australian equivalent of OBD-II [22]. In China, OBD systems have been mandatory for all vehicles since 2007 [25].

The OBD-II standard specifies a standard diagnostic connector and pin-out, electrical signalling protocols, and messaging format. It also provides a candidate list of vehicle encoded parameters to monitor which allows one to rapidly identify and remedy malfunctions within the vehicle.

OBD-II also makes it possible to read the EV SOC in real-time, after one day travel or after a full trip [26], and to send it using standard communication protocols to secondary process. At present, most OBD connectors are designed for conventional vehicles and the origin of the SOC parameter varies with different EV manufacturers. Thus, the OBD standard should practically evolve to meet applications in an EV context.

\section{EV dispatch module}

Based on the above discussion, an EV dispatch module is proposed. There are four major parts in the module: 1) the EV and owner; 2) the infrastructure; 3) the module logic; and 4) connection with other modules. The structure of the module is shown in Fig. 3. 


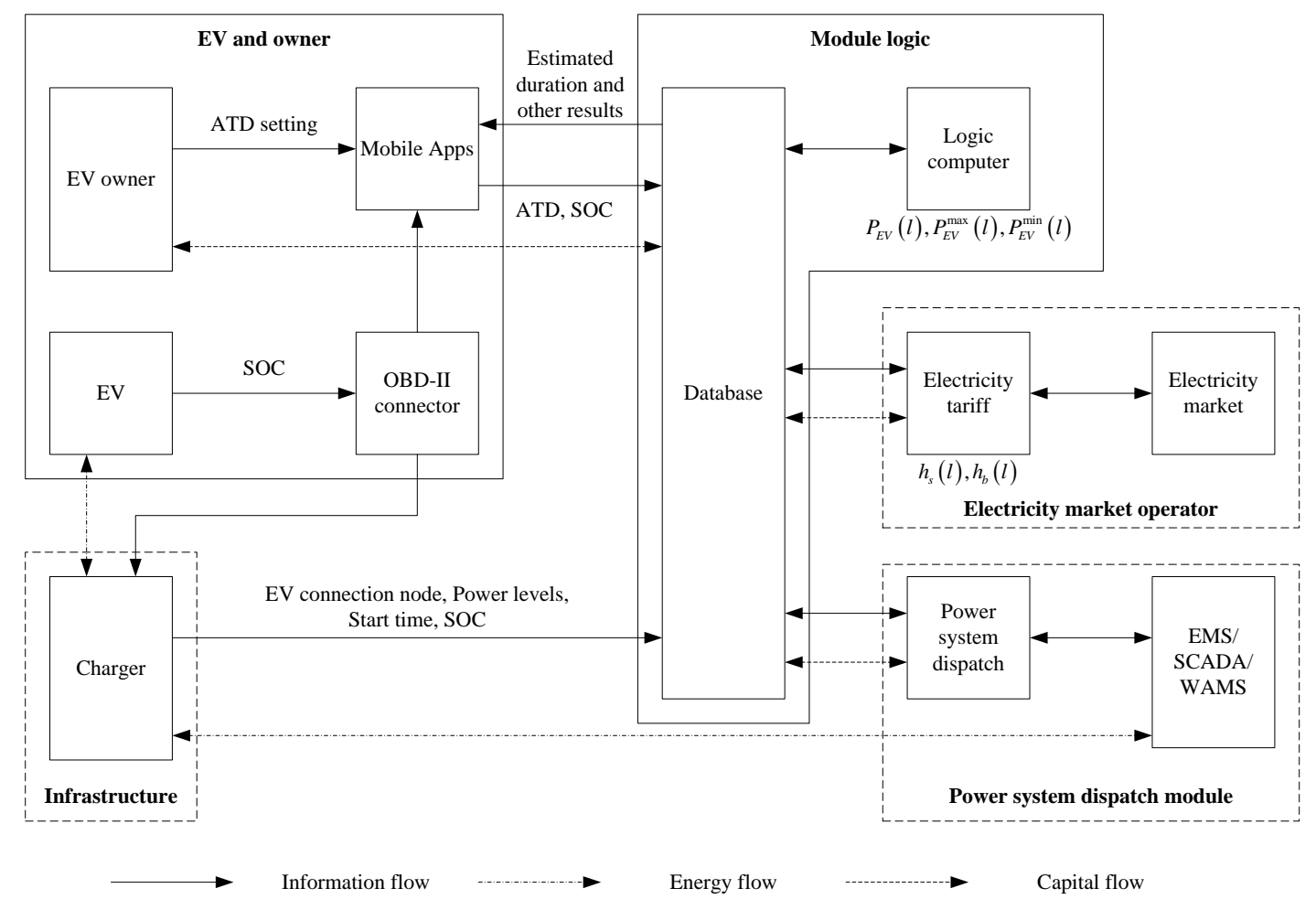

Fig. 3 The structure of a proposed EV dispatch module

\subsection{EV (and EV owner) part}

The ATD slot and initial SOC derive from this first part.

\section{1) Mobile apps}

A mobile app based on the Android, Apple or Windows (Phone) operating system (OS) was developed with a graphic I/O interface for EV owners. The app is installed on an owner mobile and provides options to set the ATD for EV charging. The ATD default value and estimated duration are available based on the initial SOC obtained from OBD-II and charging power level at the charger.

\section{2) OBD-II connector}

The SOC is obtained by the OBD-II connector and a Bluetooth communicator is installed at the connector to make it possible to transmit the SOC data to the mobile app and charger. However, the SOC data can also be transmitted to the charger by cable and thus a Bluetooth receiver is not essential in the charger.

The above information is communicated to the database in the remote logic module. The structure of the database is discussed in the logic module part.

\subsection{Infrastructure part}

\section{3) Charger}

The EV charger is installed at a fixed location and the charging power level is also fixed according to certain standards. When an EV is connected to the charger, the charger is able to identify the connection and send relevant information, such as EV connection node, power level, start time and SOC to the logic module.

\subsection{Logic module part}

\section{4) Logic computer}

In this part, $P_{E V}(l)$ is the actual EV charging power at time $l$ and $P_{E V}^{\max }(l)$ and $P_{E V}^{\min }(l)$ are the maximum and minimum adjustable limits of EV charging power. When a large number of EVs are integrated on the load side, for example in future power networks, the maximum and minimum limits should also be considered for load control and DSM.

If it is assumed that the same EV in Section 2.2 is used and Scenario 3 in Fig. 1 is considered as the discrete charging profile, then $P_{E V}(l), P_{E V}^{\max }(l)$ and $P_{E V}^{\min }(l)$ within the ATD can be shown as follows:

$$
\left[\begin{array}{c}
P_{E V} \\
P_{E V}^{\max } \\
P_{E V}^{\min }
\end{array}\right]=\left[\begin{array}{llllllllllll}
0 & 0 & 0 & 7 & 0 & 0 & 0 & 4 & 0 & 0 & 0 & 0 \\
7 & 7 & 7 & 7 & 4 & 4 & 4 & 4 & 0 & 0 & 0 & 0 \\
0 & 0 & 0 & 0 & 0 & 0 & 0 & 0 & 0 & 0 & 0 & 0
\end{array}\right] \text { (1) }
$$

where 0,4 , and 7 refer to the charging power at the start of each time interval. The unit is $\mathrm{kW}$ as shown in Fig. 2.

If the EV is charging at 21:00 and 1:00, and the minimum limit is 0 the dispatch could reduce charging demand. The maximum limit remains at the power levels specified in [17] before the EV is fully charged. It decreases to 0 after being fully charged, meaning that the dispatch could not improve the load by EV charging demand. In this case, there is only one EV and the dispatch can only choose the charging power as 0,7 or 4 . With more EVs, the dispatch will be able to change the charging power to any value.

If the EV is charging as in Scenario 2, the maximum limit remains at 7 and the minimum limit at 0 from 18:00 to 05:00. At time 05:00, both the maximum limit and minimum limit will be 4 since the EV has to be fully charged by the end of the ATD. This means in practice that the power system dispatch is not able to change the EV charging power. 
From (1) it can be seen that $P_{E V}^{\max }(l)$ and $P_{E V}^{\min }(l)$ are dependent on the EV charging profile before time $l$. Thus, an online algorithm should be proposed to calculate these two limits.

\section{5) Database}

A database is used to store the settings and calculation results. There are two major tables in the database: a static table and real-time table.

The static table stores the settings and up-to-date calculation results. The information includes: customer ID, initial SOC, real-time SOC, EV connection node, power level, estimated duration, ATD, start time, end time, real-time charging power, charging energy, electricity tariff, customer payment and maximum saving.

The real-time table stores the calculation results in the time domain. The information includes: time, real-time SOC, real-time charging power and maximum adjustable and minimum adjustable limits.

The static table is used to generate text reports to customers and for power system dispatch scheduling. The real-time table is used to provide information to power system dispatch and generate graphic reports.

\subsection{Connection with other modules}

The EV dispatch module is also able to connect to other modules in power system dispatch such as traditional dispatch and electricity market operator modules. The connection with the traditional dispatch module provides dispatch objectives which can be used to obtain the optimisation function; connection with the electricity market operator module supplies the electricity tariff, which might be changed in large-scale bidding.

\section{Online algorithm for EV dispatch module}

To offer a viable solution which considers the inherent and acknowledged difficulties of EV charging - not least in a context of a large number of connected vehicles - a multi-EV reference and single-EV real-time response (MRS2R) online algorithm for EV dispatch module is proposed. The flow chart of this algorithm is shown in Fig. 4.

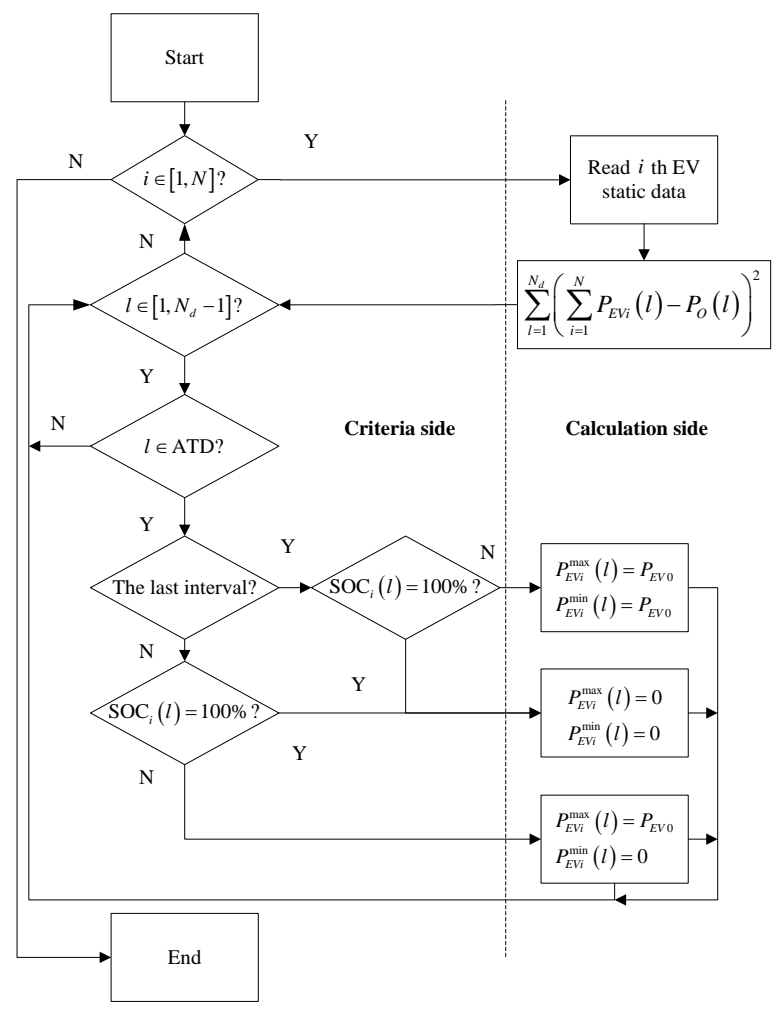

Fig. 4 Flowchart of online algorithm

In Fig. $4, N$ is the total number of EVs; $N_{d}$ is the number of equal time periods over a day period, which determines the time interval for the discretisation of the charging profile; $P_{E V}(l)$ and $P_{O}(l)$ are the EV charging power and objective power at time $l$, respectively; $\operatorname{SOC}_{i}(l), P_{E V i}^{\max }(l)$ and $P_{E V i}^{\min }(l)$ are the real-time SOC and maximum and minimum limits of the $i$ th $\mathrm{EV}$ at time $l$, respectively; $P_{E V 0}$ is the rated charging power level of $i$ th $\mathrm{EV}$; thus, $P_{E V}^{\max }(l)$ and $P_{E V}^{\min }(l)$ are the sum of maximum and minimum limits of all EVs, respectively.

The EV dispatch objective is described as the objective power $P_{O}(l)$, and the optimisation function is,

$$
\begin{aligned}
\min z & =\sum_{l=1}^{N_{d}}\left(P_{E V}(l)-P_{O}(l)\right)^{2} \\
& =\sum_{l=1}^{N_{d}}\left(\sum_{i=1}^{N} P_{E V i}(l)-P_{O}(l)\right)^{2}
\end{aligned}
$$

According to (2), the EV charging power of the $i$ th $\mathrm{EV}$ at time $l$ is obtained. The real-time $\mathrm{SOC}$ is calculated by (3),

$$
\operatorname{SOC}_{i}(l)=\operatorname{SOC}_{i}(l-1)+P_{E V i}(l) / E_{B}
$$

In (3), $E_{B}$ refers to the battery size. From (3) it can be seen that the real-time SOC at time $l$ is dependent on the SOC of the previous time interval, the battery size and the EV charging power at time $l$.

This algorithm runs during each time interval or several times ( 2 or 4 ) in one day to calculate the reference profile for all relevant EVs. When a random $\mathrm{EV}$ is connected to the grid for charging, the remaining 
$N-1$ EVs are set as constant and integrated in $P_{O}$. The online algorithm runs immediately when $N=1$ to provide optimal results for the specific EV and the results are updated in the database.

Thus, the calculation time for $N \mathrm{EVs}$ may be longer but the period for a specific EV will be much shorter. The multi-EV reference process reduces the burden for real-time calculation. Using the same algorithm for both every-interval calculation and real-time calculation minimises algorithm complexity.

\section{Case study}

\subsection{Parameters}

An initial SOC, as in [13] was adopted and a charging level of $7 \mathrm{~kW}$ [17] and the estimated duration was calculated. A battery size of $25 \mathrm{kWh}$ was assumed for the EV fleet and a fully charged duration was set to 4 hours (from 0 SOC). Thus, the ATD is assumed as a discrete uniform distribution,

$$
X \sim U\{0,20\}
$$

The start-charging time was obtained from statistics published by National Grid UK, [4] and it was assumed charging commences once a trip ends, as shown in Fig. 5. The electricity tariff is shown in Table 1, [27].

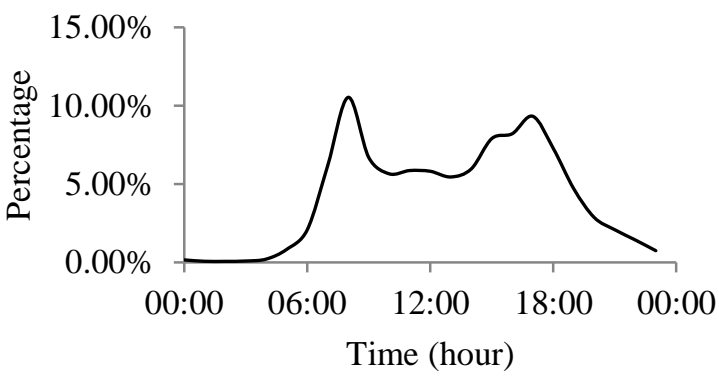

Fig. 5 EV charging start time

Table 1 Electricity tariff in different time periods

\begin{tabular}{cc}
\hline Time period & Price $(£ / \mathrm{kWh})$ \\
\hline $22: 00-06: 00$ & 0.11 \\
\hline $06: 00-08.00$ & 0.13 \\
\hline $08: 00-10: 00,18: 00-21: 00$ & 0.17 \\
\hline $10: 00-18: 00,21: 00-22: 00$ & 0.14
\end{tabular}

Based on previous studies [13], objectives can be developed for different scenarios. For simplicity, an optimal objective is considered as a flat line and only charging is considered in this study for verification of the online algorithm. Thus, (2) can be rewritten as,

$$
\begin{aligned}
\min z & =\sum_{l=1}^{N_{d}}\left(P_{E V}(l)-\bar{P}_{E V}\right)^{2} \\
& =\sum_{l=1}^{N_{d}}\left(\sum_{i=1}^{N} P_{E V i}(l)-\bar{P}_{E V}\right)^{2}
\end{aligned}
$$

where $\bar{P}_{E V}$ is the average EV charging power. Equation (5) is a quadratic programming problem which can be solved by optimisation algorithms, such as Sequence Quadratic Programming (SQP) [7, 8, 13] or the meta-heuristic algorithms such as the Genetic Algorithm (GA) [28, 29] and Particle Swarm Optimisation (PSO) [30, 31]. In this study, SQP was chosen as a least complex method to solve the above problems (in MATLAB).
By considering a minimum payment to $\mathrm{EV}$ owner scenario, an objective function can be written as,

$$
\begin{aligned}
\min z & =\sum_{l=1}^{N_{d}} P_{E V}^{M}(l) \\
& =\sum_{l=1}^{N_{d}} \sum_{i=1}^{N} h_{E V i}(l) P_{E V i}(l)
\end{aligned}
$$

where $P_{E V}^{M}$ is the payment at time $l$, and $h_{E V i}(l)$ is the electricity tariff of the $i$ th EV at time $l$. Since the electricity tariff in Table 1 is a constant, (6) is a linear programming problem which was solved using MATLAB.

\subsection{Results and discussions}

EV numbers were defined as 1, 1 hundred, 1 thousand, 10 thousand, 500 thousand and 1.5 million to test the computational efficiency of the online algorithm. 100 EVs were selected to demonstrate the detailed results of the online algorithm. The time interval was selected as 1 hour since the start time and tariff were given hourly.

\subsubsection{EVs simulation}

The ATDs of 100 EVs are shown in Fig. 6. The optimisation results are shown in Fig. 7 - Fig. 9.

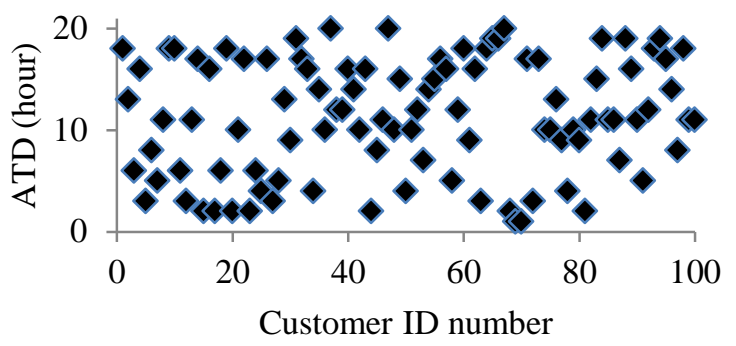

Fig. 6 ATDs of 100 EVs

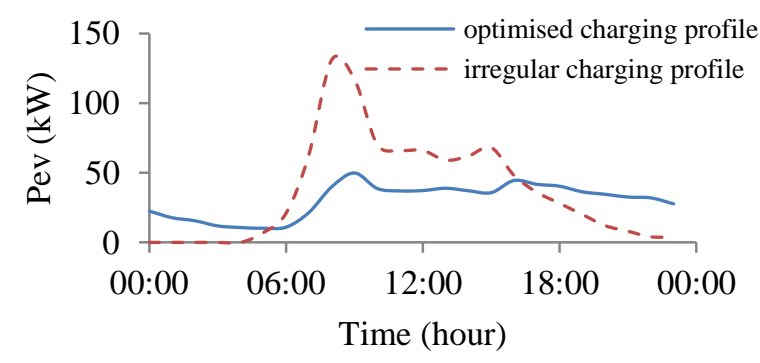

Fig. 7 Optimised charging profile for flat line

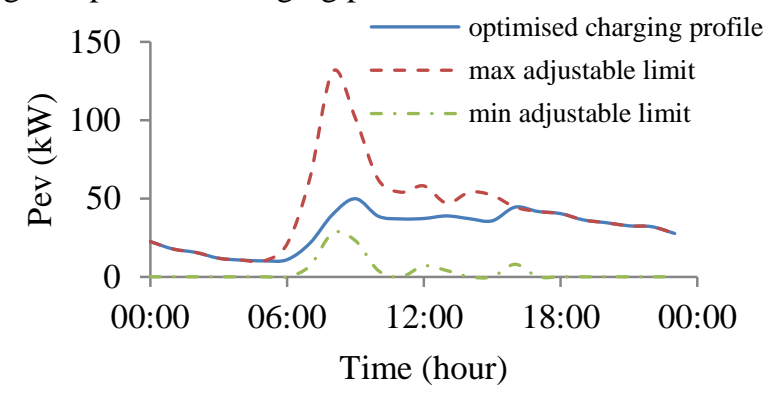

Fig. 8 Optimised charging profile for flat line with limits 


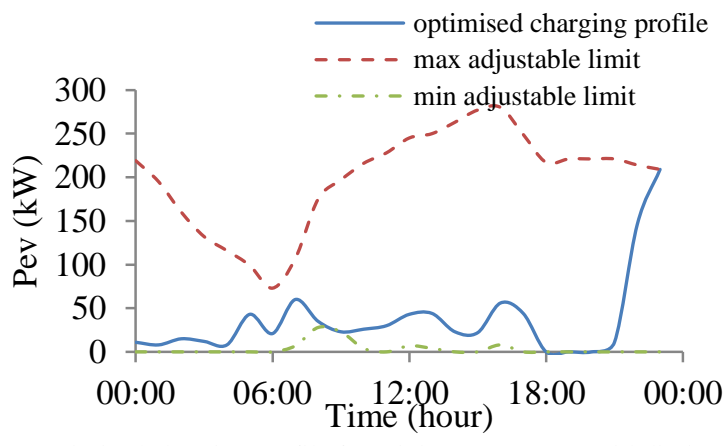

Fig. 9 Optimised charging profile for minimum payment with limits

In Fig. 7, the optimised result is not a flat line. The peak-valley difference is $49 \mathrm{~kW}$ and the standard deviation is $39.7 \%$, both as a consequence of the ATD, as in Fig. 6. Since the charging duration of each vehicle must be within its own ATD, this limits the optimisation result.

In Fig. 8, the optimised curve and the maximum limit curve overlap in some areas, meaning there is no upward adjustable margin. In power system dispatch, this means that system dispatch is unable to augment EV charging demand at that moment.

In comparison to Fig. 9 it can be concluded that when real-time EV charging demand changes (the system dispatch changes the EV charging demand), the adjustable margin also changes. In Fig. 9, the optimised curve reaches the peak at 23:00 since there are more EVs that start charging after 18:00 with short estimated charging duration and the electricity tariff after 22:00 is low.

In comparison to previous studies [7, 8, 13], two more curves are obtained in Fig. 8 and Fig. 9, which are the maximum and minimum adjustable limits. The two limits provide decision-making support for practical power system dispatch or energy market management. In Fig. 8, for instance from 6:00 to 15:00, the energy system dispatch is able to adjust the EV charging demand with the adjustable margin to participate in DSM. Furthermore, taking 9:00 in Fig. 8 as an example, the optimised demand is roughly $50 \mathrm{~kW}$, with a margin between $23 \mathrm{~kW}$ and $101 \mathrm{~kW}$, which can be considered as an EV-charging-based energy system operating reserve for 1 hour long. Therefore, in terms of real application, the system dispatch is able to increase or decrease the EV demand to benefit from energy trading and power bidding.

By using multi-objective optimisation of a flat EV charging profile and minimum payment, the peak will drop since the electricity prices from 22:00 to 06:00 are identical.

In this case, the saving for a flat line objective is $57.3 \%$ of the maximum saving; and for minimum payment, the saving is $59.4 \%$ of the maximum. The calculated saving cannot reach a maximum due to the use of an ATD. When there is a maximum payment saving, the charging duration should be within the lowest electricity tariff duration, from 22:00 to 06:00, which is possible in any realistic evening period but not possible in this case since the ATD is generated randomly by a discrete uniform distribution function in MATLAB.

An example of static and real-time tables are shown in the Appendix.
Based on this simulation, it can be concluded that the proposed online algorithm is a feasible approach to solve the EV dispatch problem. The calculation results for EV charging power and maximum and minimum adjustable limits can be part of decision-making support for power system dispatch. In conjunction with an electricity tariff, savings of each vehicle can also obtained, which could feasibly motivate EV owners to participate in active dispatch.

\subsubsection{Computational efficiency}

In this study, the simulation was executed on a PC with Intel Xeon CPU E3-1220 v3 and 16 GB RAM. The calculation time is shown in Table 4 and its trend is shown in Fig. 10

Table 4 The calculation time of the online algorithm

\begin{tabular}{ccc}
\hline EV numbers & $\begin{array}{c}\text { Min payment } \\
\text { calculation time }(\mathrm{s})\end{array}$ & $\begin{array}{c}\text { Flat line calculation time } \\
(\mathrm{s})\end{array}$ \\
\hline 1 & 0.332 & 0.390 \\
\hline 100 & 0.403 & 2.094 \\
\hline 1000 & 0.733 & 5.357 \\
\hline 10000 & 13.983 & 54.031 \\
\hline 500000 & 619.427 & 2699.71 \\
\hline 1500000 & 1854.595 & 8098.21 \\
\hline
\end{tabular}

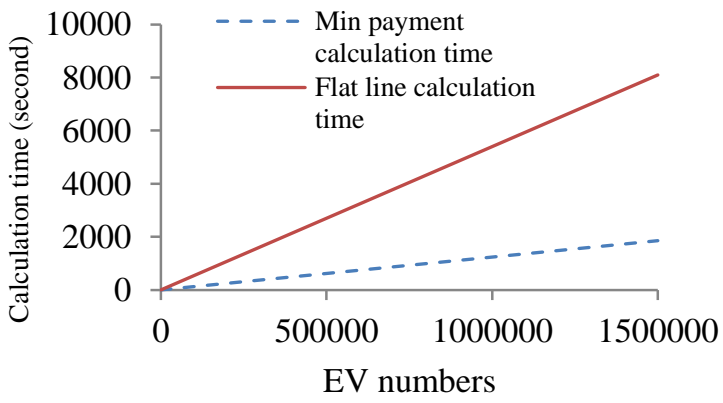

Fig. 10 Calculation time trend with respect to EV numbers

From Table 4 and Fig. 10 it is apparent that calculation time increases with a rise in EV numbers; if the EV number is $<10$ thousand, the total calculation duration is $<1$ minute.

There are two published sources which predict EV numbers in the UK (by 2020) as 500 thousand [4] and 1.5 million [32]. With 500 thousand EVs, the calculation duration is approximately 10 minutes for a minimum payment objective and roughly 45 minutes for a flat line objective. When there are 1.5 million EVs, the calculation duration is approximately 31 minutes for a minimum payment objective and 135 minutes for a flat line objective.

In $\mathrm{EV}$ dispatch, the practical objective for $\mathrm{EV}$ dispatch is usually combined with load profile $[2,7,8,10,13,30]$. In the UK, load profile is normally published every 30 minutes [33] or 15 minutes [34]. In day-ahead scheduling, a 1 hour interval is also considered $[7,13,30]$.

In this study, the computational efficiency is related to the number of EVs, discrete intervals and solution algorithm. When there are more EVs and shorter discrete intervals, the calculation time will be longer. Using more efficient meta-heuristic methods would improve computational efficiency [30]. By considering the (time) interval of the start time and tariff in this study, if the total operation time of the proposed EV dispatch module is within 1 hour, it can therefore be defined as feasible 
for calculation within each time interval.

The total operation time of the proposed EV dispatch module should also consider the mobile app time delay. The total operation time is discussed in next section.

\subsection{Mobile app time delay}

An Android mobile app was developed on the Eclipse Kepler platform to test the mobile app time delay. The communication time delay has not been included in this study. A PC (server) platform was used with Intel Xeon CPU E5-1620 0 and 8 GB RAM and the XAMPP server used as the database. The results are shown in Table 5.

Table 5 Transmission time delay

\begin{tabular}{cccc}
\hline EV numbers & $\begin{array}{c}\text { Time delay } \\
(\mathrm{s})\end{array}$ & $\begin{array}{c}\text { Total time for min } \\
\text { payment }(\mathrm{s})\end{array}$ & $\begin{array}{c}\text { Total time for } \\
\text { flat line }(\mathrm{s})\end{array}$ \\
\hline 1 & 0.175 & 0.507 & 0.565 \\
\hline 100 & 0.235 & 0.638 & 2.329 \\
\hline 1000 & 0.364 & 1.097 & 5.721 \\
\hline 10000 & 4.024 & 18.187 & 58.235 \\
\hline 500000 & 203.372 & 822.802 & 2903.085 \\
\hline 1500000 & 609.876 & 2464.471 & 8708.086 \\
\hline
\end{tabular}

In Table 5, the time delay is affected by network signal intensity, download and upload speed and CPU speed and RAM size. If a low EV penetration and limited use of the proposed app are assumed, the time delays of large-scale EVs are estimated by the message size and download speed.

From Table 5 it can be determined that when EV numbers are $<10$ thousand, the total time delay is $<1$ minute. With 500 thousand EVs, the total time delay is approximately 14 minutes for a minimum payment objective and roughly 48 minutes for a flat line objective. With 1.5 million EVs, the total delay time is approximately 41 minutes for a minimum payment objective and roughly 145 minutes for a flat line objective. Also, the total operation time for a single EV optimisation is only about $0.5-0.6$ second.

As discussed in Section 4, there are two operating processes in the proposed MRS2R online algorithm. The multi-EV reference process runs every time interval or a few times in a day. If it runs every time interval, that is 1 hour in this study, the total operation time of the algorithm should be within 1 hour. However, currently, the proposed algorithm is not feasible for 1.5 million EVs and flat line optimisation (quadratic programming) objective. It is therefore proposed that the algorithm run 2-8 times a day to work out a reference profile. Otherwise, a more powerful platform or more efficient solving algorithm should be adopted.

The single-EV real-time response process runs when there is an EV connected to the grid, which is set as a request to the server for single EV optimisation. The total operation time is only 0.5-0.6 second which is short enough for the convenience of app users.

\section{Further discussions}

\subsection{Influence of data security}

According to Fig. 3, there is capital and information flow through a mobile app. The security and privacy protection of monetary transactions and other sensitive data movement is beyond the scope of this paper. In terms of information flow, two parameters are transmitted, the initial SOC and the ATD.

Many mobile applications offer a function to remember login credentials for ease of use, which means that the same operation can also be performed by illegitimate parties once a device is lost or stolen. The developed application has to contend with the same issue that the integrity of transmitted SOC and ATD data might be compromised in theft cases unless the user prefers to type in personal credentials each time. Several countermeasures can be implemented to address this issue which includes data-driven [35] and event-driven implicit authentication [36]. The chosen authentication module is able to operate transparently in the background and monitor the trust level of a user all the time. The server can determine whether to authenticate the user or not based on this trust level.

Also, a compromise of data integrity is possible during communications, which is widely known as a man-in-the-middle (MiTM) attack [37]. A MiTM is usually conducted by the attacker in a LAN environment. Thus, strong encryption and mutual authentication techniques should be implemented to protect the integrity of data such as Transport Layer Security (TLS) and Pretty Good Privacy (PGP).

By considering the optimised profile in Fig. 7, when the SOC and ATD data are secured and correct, an optimised result can be obtained.

If the SOC data is changed to be smaller than its actual value, the battery might be over-charged after the SOC reaches $100 \%$, which can be harmful to $\mathrm{EV}$ batteries, unless protected. If the SOC data is changed to be larger than its actual value, the EV charging will be incomplete, which would compromise owner requirements.

If the ATD data is changed to be larger than the setting value, optimisation might be more flexible with greater savings. However, owners are not guaranteed to get their car fully charged when next trip starts. If ATD data is changed to be smaller than the setting value, dispatch flexibility will be suppressed. If the duration is set as only one interval, the charging power may be very high, resulting in negative effect on the load curve.

If the integrity of the SOC and ATD are both compromised, for example $0 \%$ SOC and only 1 interval for the ATD, this means all connected EVs will receive $1 \mathrm{C}$ [32] current charging at the same time. In particular, if the Northern Ireland (NI) power system load profile [33] in Fig. 11 is considered with all EVs charging at 17:00, the load peak will be substantially increased.

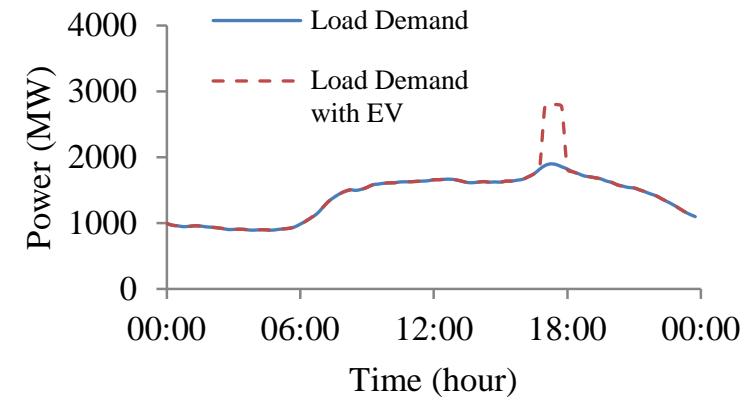

Fig. 11 NI winter load demand

In Fig. 11, the peak-valley difference increase around 90.4\% from 1006.2 MW to $1916.18 \mathrm{MW}$, which would aggravate power flow as well as limit generator ramping. Moreover, in this situation, power system dispatch would be unable to adjust $\mathrm{EV}$ power and payment from EV 
owners, which would increase significantly.

From the above discussion, it can be concluded that when the SOC data and the ATD data are compromised, negative effects can occur which may ultimately lead to impacts on profitability and revenue for EV stakeholders. Thus, the data security of mobile apps in the EV dispatch module must be strictly considered.

\subsection{EV dispatch trend and commercial impact}

With limited EV penetration and a largely undeveloped market at present, EV owners are typically less interested in any discharging mode unless compensation applies [38]. Equally, owner participation in scheduled charging is also of limited interest if no financial incentives are offered or adopted based on tariff changes [39-42], with a preference for immediate (and uncomplicated) once an EV is connected - which might be then considered as irregular charging.

From the previous case study, it has been demonstrated that the proposed EV dispatch module is able to provide real-time savings based on knowledge of charging profile, ATD and tariff. Parameters may be sent to EV owners instantaneously and they are able to check results all the time. Thus such results could not only encourage participation in EV dispatch, but also help power system dispatch in decision-making support.

As with business models proposed for vehicle-to-grid (V2G) in [43], there are three prospective models for EV dispatch, depending on who is in charge of EV dispatch.

The first mode is the EV owner (fleet operator) mode. In this mode, EV usage for transportation and charging demand profile are both managed. However, owners and the utilities have to come to an agreement for electricity bidding in the electricity market. This mode is suitable for public service vehicles such as civil service vehicles, buses, post office vehicles, and electric utility vehicles since these EV fleets are more controllable.

The second mode is a utility mode. Home based charging is one of the most common forms of this mode and is quite common at present in many countries. Power system dispatch in utilities manages the charging demand and the EV owner simply charges the EV, as with other electric equipment such as mobile phones or laptops.

The last mode is a third-party mode, which is derived from the previous two. As discussed in Section 5.2, with large numbers of EVs and complicated optimal objectives, computational efficiency may be compromised using limited computer platforms. Hence it is not feasible to ask owners or a utility department to manage. In this mode, an independent third-party with powerful computational resources would be preferable. In practice, such parties might include automobile manufacturers, service organisations, battery companies or distributors, cell phone network providers, insurance companies or a distributed generation company.

\section{Conclusions and future work}

This paper proposes a four-part EV module for power system dispatch as a primary component in $\mathrm{EV}$ connection and integration and as an integral element in demand-side participation and management. OBD-II and mobile apps are considered which incorporates EV owner participation and accurate knowledge of actual initial SOC and ATD parameters. The maximum and minimum adjustable limits of EV charging demand is calculated by the proposed MRS2R online algorithm.

The EV dispatch module has been evaluated using numerical case studies. A discussion of computational efficiency and the mobile-app time delay reveals that the proposed algorithm is feasible for online and real-time operation, though practically limited to $<1$ million EVs using flat-line objective optimisation. It is proposed that a multi-EV reference process could be executed 2-8 times a day to overcome shortcomings in computer technology or until efficient algorithms are adopted for $>1$ million EVs. The influence of data security has also been discussed in the case study.

Future EV dispatch needs to involve close participation of EV owners to achieve flexibility. By providing financial incentives and charging limits in real-time, owners can be encouraged as active stakeholders in demand-side management. The paper has also considered three potential business models for commercial application which may assist dissemination of the proposed approach.

The paper proposes a new approach which embraces real-time, EV owner-interaction. The benefits of the work presented in this paper are timely and the primary contributions are perceived of particular importance to EV manufacturers, owners, power system operators and business developers. The work is also considered valuable for the development of EV infrastructure particularly demand-side energy solutions and diverse micro-grid technologies - and dissemination of viable technical methods to support contributory technologies, including reliable and secure communications networks, domestic Smart Meters and enhanced Smartphone applications.

In future work, research will involve greater inclusion of OBD-II and powerful mobile apps which are adaptable to different types of EVs. Moreover, additional work will include investigation of third-party business models to operate the proposed module in real EV fleets and provide decision-making support for power system dispatch to meet charging demand and support demand response and management.

\section{Appendix}

The example static table is shown in Table A1. The real-time EV SOC is shown in Fig. A1.

Table A1 Static table in EV dispatch

\begin{tabular}{|c|c|c|c|c|c|c|c|c|c|c|c|c|}
\hline ID & SOCi & $\mathrm{SOCr}$ & Pev0 & estd & ATD & stt & rstt & redt & Wev & ptot & save & $\operatorname{maxs}$ \\
\hline units & $\%$ & $\%$ & $\mathrm{~kW}$ & $\mathrm{~h}$ & $\mathrm{~h}$ & 1 & 1 & 1 & $\mathrm{kWh}$ & pence & pence & pence \\
\hline 1 & 0 & 0 & 7 & 4 & 18 & 5 & 5 & 23 & 25 & 303 & 24 & 52 \\
\hline 2 & 28 & 28 & 7 & 3 & 13 & 6 & 6 & 11 & 18 & 238 & 12 & 52 \\
\hline 3 & 28 & 28 & 7 & 3 & 6 & 6 & 6 & 12 & 18 & 238 & 12 & 52 \\
\hline 4 & 28 & 28 & 7 & 3 & 16 & 7 & 7 & 23 & 18 & 233 & 45 & 80 \\
\hline 5 & 28 & 28 & 7 & 3 & 3 & 7 & 7 & 10 & 18 & 278 & 0 & 80 \\
\hline
\end{tabular}




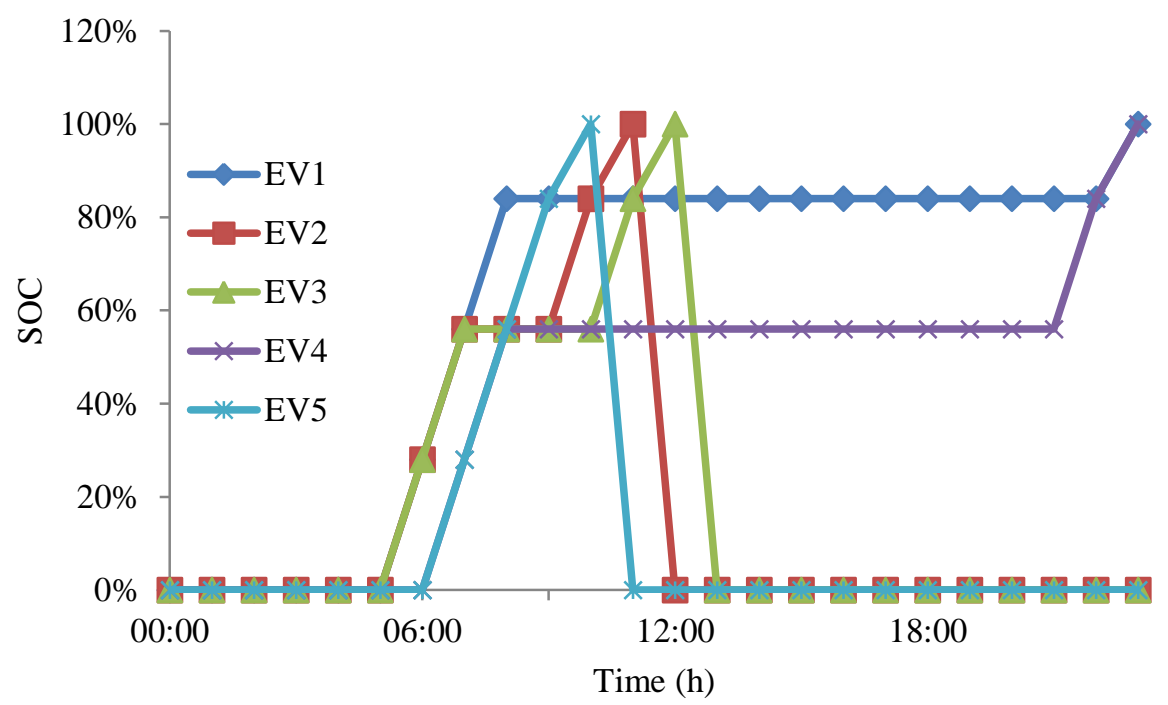

Fig. A1 Real-time EV SOC of EV 1, 2, 3, 4, and 5

In Table A1: column 1 is the customer ID; column 2 the initial SOC; column 3 the real-time SOC; column 4 the power level; column 5 the estimated duration; column 6 the ATD; column 7 the start time of ATD; column 8 the real start time; column 9 the real end time; column 10 the energy consumption; column 11 the total payment; column 12 the payment saving for this time; and column 13 is the maximum saving.

Fig. A1 only shows the real-time SOC of $5 \mathrm{EVs}$ corresponding to the 5 examples in Table A1.

\section{Acknowledgments}

The authors would like to acknowledge the support of the EPSRC UK-China joint research consortium (EP/F061242/1), the Science Bridge Award (EP/G042594/1), Chinese Scholarship Council and Queen's University Belfast, UK.

\section{Reference}

[1] P Kundur. Power System Stability and Control. New York: McGraw Hill 1994.

[2] Z Yang, K Li, Q Niu, et al. A self-learning TLBO based dynamic economic/environmental dispatch considering multiple plug-in electric vehicle loads. J Mod Power Syst Clean Energy 2014; 2(4): 298-307.

[3] D Madzharov, E Delarue, W D'haeseleer. Integrating electric vehicles as flexible load in unit commitment modeling. Energy 2014; 65: 285-294.

[4] National Grid. UK Future Energy Scenarios; 2014. Available: <http://www2.nationalgrid.com/UK/Industry-information/Future -of-Energy/Future-Energy-Scenarios/> [Accessed: 22/06/15]

[5] $\mathrm{X} \mathrm{Hu}, \mathrm{N}$ Murgovski, LM Johannesson, et al. Optimal dimensioning and power management of a fuel cell/battery hybrid bus via convex programming. IEEE/ASME Trans Mechatronics 2015; 20(1): 457-468.

[6] Y Zou, X Hu, H Ma, et al. Combined State of Charge and State of Health estimation over lithium-ion battery cell cycle lifespan for electric vehicles. J Power Sources 2015; 273: 793-803.

[7] P Zhang, K Qian, C Zhou, et al. A methodology for optimization of power systems demand due to electric vehicle charging load. IEEE Trans Power Syst 2012; 27(3): 1628-1636.

[8] H Cai, W Du, XP Yu, et al. Day-ahead Optimal Charging/Discharging Scheduling for Electric Vehicles in Micro-Grids. In: IET Renewable Power Generation Conference 2013; 2013. p. $1-4$

[9] R Sioshansi. Modeling the impacts of electricity tariffs on plug-in hybrid electric vehicle charging, costs, and emissions. Operations Research 2012; 60(2): 1-11.

[10] Jun Yang, Lifu He, Siyao Fu. An improved PSO-based charging strategy of electric vehicles in electrical distribution grid. Appl
Energy 2014; 128: 82-92.

[11] Klaas De Craemer, Stijn Vandael, Bert Claessens, et al. An Event-Driven Dual Coordination Mechanism for Demand Side Management of PHEVs. IEEE Trans Smart Grid 2014; 5(2): 751-760.

[12] Chiao-Ting Li, Changsun Ahn, Huei Peng, et al. Synergistic Control of Plug-In Vehicle Charging and Wind Power Scheduling. IEEE Trans Power Syst 2013; 28(2): 1113-1121.

[13] Bowen Zhou, Tim Littler, Aoife Foley. Electric Vehicle Capacity Forecasting Model with Application to Load Levelling. In: IEEE PES General Meeting 2015; 2015. p. 1-5.

[14] Joakim Munkhammar, Joakim Widén, Jesper Rydén. On a probability distribution model combining household power consumption, electric vehicle home-charging and photovoltaic power production. Appl Energy 2015; 142: 135-143.

[15] Myriam Neaimeh, Robin Wardle, Andrew M Jenkins, et al. A probabilistic approach to combining smart meter and electric vehicle charging data to investigate distribution network impacts. Appl Energy 2015; 157: 688-698.

[16] PG Leahy, AM Foley. Impact of weather on electric vehicle performance. In: Proc Irish Transport Research Network Conf 2011; 2011. p. 1-4

[17] IEC Standard. Plugs, socket-outlets, vehicle connectors and vehicle inlets - Conductive charging of electric vehicles - Part 2 : Dimensional compatibility and interchangeability requirements for a.c. pin and contact-tube accessories, IEC 62196-2; 2011.

[18] Statutory Rules of Northern Ireland. The Parking Places on Roads (Electric Vehicles) (Amendment No. 3) Order (Northern Ireland) 2014; $2014 . \quad$ Available: <http://www.ecarni.com/parking-places-on-roads-electric-vehicl es-amendment-no-3-order> [Accessed 22/06/15]

[19] E-car NI. Mobile phone app; 2015. Available: < http://www.ecarni.com/mobile-phone-app> [Accessed: 22/06/15]

[20] Guido Benetti, Maurizio Delfanti, Tullio Facchinetti, et al. Real-Time Modeling and Control of Electric Vehicles Charging Processes. IEEE Trans Smart Grid 2015; 6(3): 1375-1385.

[21] Mostafa F. Shaaban, Muhammad Ismail, Ehab F. El-Saadany, et al. Real-Time PEV Charging/Discharging Coordination in Smart Distribution Systems. IEEE Trans Smart Grid 2014; 5(4): 1797-1807.

[22] Wikipedia. On-board diagnostics; 2015. Available: <http://en.wikipedia.org/wiki/On-board_diagnostics\#cite_note-2> [Accessed: 22/06/15].

[23] ISO Standard. Road vehicles -- Diagnostic communication over Controller Area Network (DoCAN) -- Part 4: Requirements for emissions-related systems, ISO 15765-4:2011; 2011.

[24] European Standard. European Emission Standards Directive 98/69/EC of the European Parliament and of the Council, Directive 98/69/EC; 1998.

[25] China Standard. Limits and measurement methods for emissions from light-duty vehicles (III, IV), GB 18352.3-2005; 2005.

[26] EVtools.info. Chevy Volt OBD2 CAN Data; 2015. Available: <http://www.evtools.info/ChevyVoltOBD2CAN.html> [Accessed: 22/06/15] 
[27] SY Wang, JL Yu. Optimal sizing of the CAES system in a power system with high wind power penetration. Int $\mathrm{J}$ Electr Power Energy Syst 2012; 37(1): 117-125.

[28] Swagatam Das, Ponnuthurai Nagaratnam Suganthan. Differential Evolution: A survey of the State-of-the-Art. IEEE Trans Evol Comput 2011; 15(1): 4-31.

[29] L Zhang, Z Wang, X Hu, et al. A comparative study of equivalent circuit models of ultracapacitors for electric vehicles. J Power Sources 2015; 274: 899-906.

[30] Z Yang, K Li, A Foley, et al. Optimal scheduling methods to integrate plug-in electric vehicles with the power system: a review. In: 19th World Congress of the International Federation of Automatic Control. IFAC, 2014; 2014. p. 8594-8603.

[31] Yamille del Valle, Ganesh Kumar Venayagamoorthy, Salman Mohagheghi, et al. Particle Swarm Optimization: Basic Concept, Variants and Applications in Power Systems. IEEE Trans Evol Comput 2008; 12(2): 171-195.

[32] BW Zhou, T Littler, HF Wang. The impact of vehicle-to-grid on electric power systems: a review. In: IET Renewable Power Generation Conference 2013; 2013. p. 1-4.

[33] National Grid. Historical Demand Data; 2015. Available: $<$ http://www2.nationalgrid.com/UK/Industry-information/Electri city-transmission-operational-data/Data-explorer/> [Accessed: 22/06/15].

[34] EirGrid. System Demand; 2015. Available: <http://www.eirgrid.com/operations/systemperformancedata/syst emdemand/> [Accessed: 22/06/15].

[35] Hilmi Gunes Kayacik, Mike Just, Lynne Baillie, et al. Data Driven Authentication: On the Effectiveness of User Behaviour Modelling with Mobile Device Sensors. In: Proc the Third Workshop on Mobile Security Technologies (MoST) 2014; 2014. p. 1-10.

[36] Feng Yao, Suleiman Y Yerima, BooJoong Kang, et al. Event-driven Implicit Authentication for Mobile Access Control. In: 9th International Conference on Next Generation Mobile Applications, Services and Technologies; 2015. p. 1-8.

[37] Mayank Agarwal, Santosh Biswas, and Sukumar Nandi. Advanced Stealth Man-in-The-Middle Attack in WAP2 Encrypted Wi-Fi Network. IEEE Communications Letters 2015; 19(4): 581-584.

[38] Becky Gough. The Limitations and Potential Barriers to Wide-Scale Vehicle-to-Grid in the UK?; 2015. Available: $<\mathrm{http}: / /$ electricvehicle.ieee.org/2015/08/28/the-limitations-and-p otential-barriers-to-wide-scale-vehicle-to-grid-in-the-uk/> [Accessed: 10/09/15]

[39] AG Boulanger, AC Chu, S Maxx, et al. Vehicle electrification: Status and issues. Proc IEEE 2011; 99(6): 1116-1138.

[40] M Kezunovic, JD McCalley, TJ Overbye. Smart grids and beyond: Achieving the full potential of electricity systems. Proc IEEE 2012; 100: 1329-1341.

[41] J Mullan, D Harries, T Bräunl, et al. The technical, economic and commercial viability of the vehicle-to-grid concept. Energy Policy 2012; 48: 394-406.

[42] Elham Azadfar, Victor Sreeram, David Harries. The investigation of the major factors influencing plug-in electric vehicle driving patterns and charging behaviour. Renewable and Sustainable Energy Reviews 2015; 42: 1065-1076.

[43] W Kempton, J Tomić. Vehicle-to-grid power implementation: From stabilizing the grid to supporting large-scale renewable energy. J Power Sources 2005; 144(1): 280-294. 